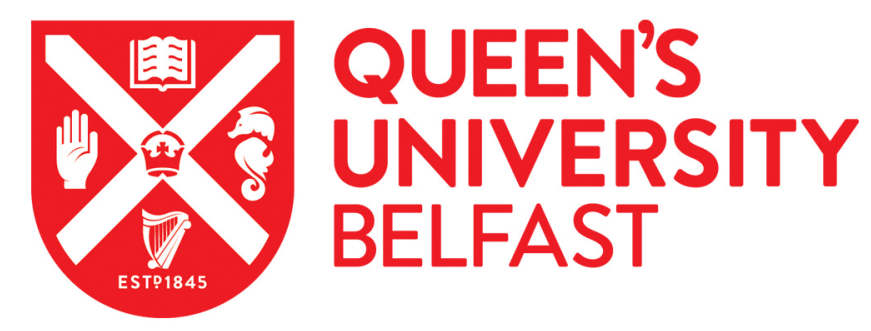

\title{
Mechanical and Thermal Properties of Polyphenylene Sulfide/Multiwalled Carbon Nanotube Composites
}

Jiang, Z., Hornsby, P., McCool, R., \& Murphy, A. (2012). Mechanical and Thermal Properties of Polyphenylene Sulfide/Multiwalled Carbon Nanotube Composites. Journal of Applied Polymer Science, 123(5), 2676-2683. https://doi.org/10.1002/app.34669

Published in:

Journal of Applied Polymer Science

Document Version:

Peer reviewed version

Queen's University Belfast - Research Portal:

Link to publication record in Queen's University Belfast Research Portal

Publisher rights

Copyright 2011 Wiley Periodicals, Inc. This work is made available online in accordance with the publisher's policies. Please refer to any applicable terms of use of the publisher.

\section{General rights}

Copyright for the publications made accessible via the Queen's University Belfast Research Portal is retained by the author(s) and / or other copyright owners and it is a condition of accessing these publications that users recognise and abide by the legal requirements associated with these rights.

Take down policy

The Research Portal is Queen's institutional repository that provides access to Queen's research output. Every effort has been made to ensure that content in the Research Portal does not infringe any person's rights, or applicable UK laws. If you discover content in the Research Portal that you believe breaches copyright or violates any law, please contact openaccess@qub.ac.uk. 


\title{
Mechanical and thermal properties of polyphenylene
}

\section{sulphide/multi-walled carbon nanotube composites}

\author{
Zhenyu Jiang, Peter Hornsby, Rauri McCool, Adrian Murphy \\ School of Mechanical and Aerospace Engineering, Queen's University Belfast, Belfast, BT9 \\ $5 \mathrm{AH}, \mathrm{UK}$
}

\begin{abstract}
Polyphenylene sulphide (PPS)/multi-walled carbon nanotube (MWCNT) composites were prepared using a melt-blending procedure combining twin-screw extrusion with centrifugal premixing. A homogeneous dispersion of MWCNTs throughout the matrix was revealed by scanning electron microscopy for the nanocomposites with MWCNT contents ranging from 0.5 to $8.0 \mathrm{wt} \%$. The mechanical properties of PPS were markedly enhanced by the incorporation of MWCNTs. Halpin-Tsai equations, modified with an efficiency factor, were used to model the elastic properties of the nanocomposites. The calculated modulus showed good agreement with the experimental data. The presence of the MWCNTs exhibited both promotion and retardation effects on the crystallization of PPS. The competition between these two effects results in an unusual change of the degree of crystallinity with increasing MWCNT content.
\end{abstract}

Page 1 of 20 


\section{Introduction}

Carbon nanotube (CNT)-reinforced polymer composites have gained increasing interest since the 1990's. ${ }^{1,2}$ The exceptional mechanical, electrical and thermal properties of CNTs, together with their nano-scale dimensions and high aspect ratios, make them superior additives for mechanical reinforcement of structural composites or fabrication of conductive polymers. ${ }^{3}$ It has been extensively reported that the elastic modulus, strength and fracture toughness of various polymers can be significantly enhanced by the incorporation of CNTs at modest loadings. ${ }^{4}$ Furthermore, integration of CNTs into traditional fibre-reinforced composites have demonstrated further improved mechanical performance, especially interlaminar and through-thickness properties. $^{5,6}$

Polyphenylene sulphide (PPS) is a semi-crystalline aromatic polymer which possesses excellent mechanical performance, good stability at elevated temperature, outstanding chemical resistance and inherent flame retardancy. In addition to these advantages, the relatively low material cost and good processability has made PPS a highly competitive engineering material among current high performance thermoplastics. It was estimated that the global demand of PPS will maintain an annual growth rate of approximately $10 \%{ }^{7}$ However, high-end applications of PPS are still limited due to its inferior mechanical properties (strength and toughness) in comparison to the other commonly used aromatic thermoplastics, such as polyetheretherketone (PEEK) and polyetherimide (PEI). In order to overcome those deficiencies, considerable research effort has been directed towards PPS-matrix composites. Modification of PPS with nanoparticles ${ }^{8-16}$, nanoclay ${ }^{17-19}$ and nanotubes ${ }^{20-24}$ have undergone rapid development in recent years. It was reported that the mechanical, thermal and wear properties of PPS can be significantly improved via nano-scale additives. 
There have been only limited publications concerned with CNT-reinforced PPS composites so far. Addition of multi-walled carbon nanotubes (MWCNTs), via melt-blending ${ }^{22-24}$ or direct powder-mixing followed by compression moulding ${ }^{20,21}$, was found to demonstrate a remarkable reinforcing effect on the mechanical properties of PPS. Yu et al ${ }^{22}$ achieved increases of $35 \%$ and $206 \%$ in Young's modulus and tensile strength respectively by adding 7 wt $\%$ MWCNTs into PPS. Wu et al $^{23}$ also observed that Young's modulus and tensile strength were increased by $86 \%$ and 209\% in PPS with the same MWCNT loading. However, it was notable that the mechanical properties of the unfilled PPS in those studies were below the normal levels. For instance, unfilled PPS usually possesses a tensile modulus of 2.6-3.8 GPa and a tensile strength of 65-86 MPa. ${ }^{25,26}$ In contrast, the modulus and strength of unfilled PPS reported by the two groups were only 1.4-1.8 GPa and 20-26 MPa, respectively. Even though the tensile strength of the PPS/MWCNT composites was greatly increased, it is still lower than that of commercially available unfilled PPS.

Contradictory observations of thermal properties of PPS/MWCNT composites were also shown in the published work. It was found that the peak crystallization temperature $\left(T_{c}\right)$ of PPS was monotonically raised with the addition of MWCNTs. ${ }^{23,24}$ However, another study demonstrated that $T_{c}$ of PPS/MWNCT was $5-10{ }^{\circ} \mathrm{C}$ lower than that of unfilled PPS. ${ }^{22}$ One group reported an increase in peak melting temperature $\left(T_{m}\right)$ caused by the presence of MWCNT, ${ }^{23}$ whereas others observed that $T_{m}$ of PPS/MWCNT composites exhibited a gradual decrease. ${ }^{22,24}$ The existing inconsistencies necessitate the further investigations to achieve an unambiguous image of the properties of PPS/MWCNT composites.

This paper addresses two objectives. The first concerns the preparation of PPS/MWCNT composites. A processing strategy that combines melt-blending with pre-mixing of the additive 
powder is applied to achieve a homogeneous dispersion of MWCNTs within the PPS matrix. The second involves an exploration of the effects that MWCNTs impose on the mechanical and thermal properties of PPS. The fundamental role of nanotubes in the reinforcement mechanism is discussed according to the results from experimental finding and theoretical analysis. The influence of MWCNTs on the crystallization and melt behaviour of PPS was studied based on differential scanning calorimetry (DSC) characterization.

\section{Experimental}

\subsection{Materials}

The matrix material was a commercial PPS product (Fortron 0214, Ticona) suitable for extrusion and injection moulding, supplied in powder form with an average particle size of 300 $\mu \mathrm{m}$.

Thin MWCNTs (NC 7000), produced via catalytic chemical vapour deposition, were provided by Nanocyl SA. According to the supplier's datasheet, this type of MWCNT has an average diameter of $9.5 \mathrm{~nm}$ and length of $1.5 \mu \mathrm{m}$. However, Morcom et al ${ }^{27}$ determined using transmission electron microscopy (TEM) and scanning electron microscopy (SEM) that the average diameter and length for this product were $10.4 \mathrm{~nm}$ and $0.7 \mu \mathrm{m}$ respectively. Their measurement of 100 MWCNTs gave a distribution of CNT outside diameters ranging from $5 \mathrm{~nm}$ to $23 \mathrm{~nm}$, as listed in Table 1. Based on their study, physical parameters of NC 7000 MWCNTs can be estimated using the method proposed by Thostenson and Chou. ${ }^{28}$ Assuming that the graphitic layers of the nanotube shell have the same density as fully dense graphite $\left(\rho_{g}=2250\right.$ $\mathrm{kg} / \mathrm{m}^{3}$ ), the density of individual MWCNT $\left(\rho_{C N T}\right)$ can be calculated by: 


$$
\rho_{C N T}=\frac{\rho_{g}\left(d^{2}-d_{i}^{2}\right)}{d^{2}}
$$

where $d$ and $d_{i}$ are outside and inside diameters of the nanotube, respectively. In this work, the value obtained from Ref. ${ }^{27}$ were used, namely $\rho_{C N T}=1850 \mathrm{~kg} / \mathrm{m}^{3}$. The effective modulus of nanotube $\left(E_{f}\right)$ can be estimated according to the assumptions that the outer layer of the MWCNT carries nearly the entire load transferred from the matrix and the outer wall acts as an effective solid fibre:

$$
E_{f}=\frac{4 t}{d} E_{C N T}
$$

where $t$ is the thickness of the outer layer $(\sim 0.34 \mathrm{~nm}),{ }^{28}$ and $E_{C N T}$ represents the elastic modulus of the nanotube $(\sim 1.0 \mathrm{TPa}) .{ }^{29}$ Equation $(2)$ is valid when $(t / d)<0.25$.

\subsection{Preparation of PPS/MWCNT composites}

All materials were dried in an electrical dryer with air circulation at $110^{\circ} \mathrm{C}$ for 6 hours before processing in order to avoid the disturbance of humidity. The PPS powders and MWCNTs were then pre-mixed using a high-speed centrifugal mixer (Rondol Technology Ltd) at $3000 \mathrm{rpm}$ for $30 \mathrm{sec}$, as illustrated in Figure 1. This process effectively eliminated the large agglomerates of nanotubes, which were clearly visible to the naked-eye before the pre-mixing, and achieved a uniform distribution of MWCNTS coated on the PPS powder. The mixtures were melt-blended in a HAAKE twin-screw extruder (Rheomex PTW 16) equipped with $16 \mathrm{~mm}$ diameter corotating screws. The processing temperatures were maintained at 300,310 and $290{ }^{\circ} \mathrm{C}$ along the extruder barrel. And the rotation speed was held at $150 \mathrm{rpm}$. Five PPS/MWCNT compounds with CNT weight fractions of $0.5,1.0,2.0,4.0$ and $8.0 \%$ were prepared in this study. Unfilled PPS was also processed through the same extrusion procedure for reference purpose. 
Mechanical tensile and flexural test specimens were prepared on an Arburg Allrounder 320S 500-150 injection moulding machine, with barrel temperature profile ranging from 290 to $320{ }^{\circ} \mathrm{C}$. The mould temperature was set at $70{ }^{\circ} \mathrm{C}$ using a water circulation temperature controller.

\subsection{Characterization and test}

Morphological characterization of the PPS/MWCNT composites was conducted on a field emission environmental SEM (JEOL JSM-6500F) using an accelerating voltage of $5 \mathrm{kV}$.

The mechanical performance of the nanocomposites was evaluated through tensile and flexural tests undertaken on a universal tester (Instron 4411), following ASTM D 638 and D 790. In all tests, the distance travelled by the upper crosshead was used to calculate the breaking strain of the samples, which were out of the measurable range of the available extensometer.

DSC was conducted on a Perkin-Elmer DSC-6 to analyze the non-isothermal crystallization and melt behaviour of PPS and its nanocomposites. Samples with a typical mass of approximately $10 \mathrm{mg}$ were sealed in aluminium pans and heated from 25 to $310{ }^{\circ} \mathrm{C}$, then held at this temperature for 5 minutes to eliminate and material thermal history. The samples were then cooled and heated again in the same temperature range at a scanning rate of $5{ }^{\circ} \mathrm{C} / \mathrm{min}$. The degree of crystallinity $\left(\chi_{c}\right)$ was calculated from the DSC data according to the following equation:

$$
\chi_{c}=\frac{\Delta H}{\Delta H_{f}\left(1-W_{f}\right)}
$$

where $W_{f}$ denotes the weight fraction of the MWCNTs. $\Delta H$ represents the enthalpy of fusion, and $\Delta H_{f}$ is the enthalpy of fusion for $100 \%$ crystalline PPS, taken as $112 \mathrm{~J} / \mathrm{g} .{ }^{30}$ The property values obtained here represent an average of the results for tests run on two specimens. 


\section{Results and discussion}

\subsection{Dispersion of MWCNTs in PPS matrix}

Figure 2 shows the SEM micrographs of the cryo-fractured surface of PPS filled with 0.5, 2.0 and $8.0 \mathrm{wt} \%$ MWCNTs, taken at $\times 20,000$ magnification. It can be seen that the MWCNTs are homogeneously distributed as individual nanotubes or slightly entangled agglomerates, with good bonding to the PPS matrix. The length of the nanotubes varies from hundreds of nanometers to several microns. It is noteworthy that the diameter of the nanotubes in these SEM micrographs is greater than the values (around 10-20 nm) provided in product datasheet or measured by other researchers using TEM. ${ }^{27,31}$ The reason for this is likely that only nanotubes with large diameters can be discerned using the SEM micrographs, with smaller nanotubes easily concealed by gold coating or matrix wrapping, which are generally in the order of tens of nanometers.

\subsection{Mechanical properties of PPS/MWCNT composites}

The mechanical properties of PPS nanocomposites were improved markedly in comparison to unfilled PPS (Table 2). The Young's modulus $(E)$ and tensile strength were increased by $36 \%$ and $12 \%$ respectively in PPS filled with $8 \mathrm{wt} \%$ MWCNT. As shown in Figure 3, a turning point appeared around 1-2 wt \%, indicating that the nanotubes were less effective in reinforcement at higher CNT loadings. The reinforcement magnitude $\left(d E / d V_{f}\right.$, where $V_{f}$ is the volume fraction of CNTs) of melt-processed MWCNT-reinforced thermoplastics was found to vary from $\sim 2.4$ to $\sim 64 \mathrm{GPa}^{4,}{ }^{32}$ In our study, $d E / d V_{f}$ reached a high level $(\sim 60.5 \mathrm{GPa})$ at low CNT loading $(0.5$ wt $\%$ ) but gradually fell to a modest level (15.8 GPa) when CNT content was above $1.0 \mathrm{wt} \%$. This may be due to the increased level of poor dispersion and severer entanglement of nanotubes 
at increased CNT loadings. The breaking strain of PPS nanocomposites exhibited a monotonic decrease with increasing CNT content. With $8 \mathrm{wt} \%$ MWCNT it was reduced by $46 \%$ compared to that of unfilled PPS. The results may reflect the confining effect of MWCNTs on the movement of polymer chains at room temperature. Analogous enhancing effect was also found in flexural properties. The flexural modulus and strength of PPS nanocomposites were increased by up to $20 \%$ and $14 \%$ respectively.

The reinforcing effect of MWCNT on the strength of polymers varied in a wide range in different work. ${ }^{43}$ Incorporation of CNT into high performance thermoplastics was found to demonstrate less dramatic enhancement in strength than that expected by theory or that observed in the nanocomposites based on thermosets and common thermoplastics. Only $12-14 \%$ of increase in tensile and flexural strength was observed in our work. The addition of MWCNT into PEEK was also reported to yield a lower increase, up to $5.7 \%$ and $7.5 \%$, in tensile and flexural strength respectively. ${ }^{34}$ The possible reasons may relate to the difficulties in mixing and wetting of CNTs in those high performance thermoplastics, which have a high melt viscosity. The relatively poor CNT dispersion and weak interface between CNT and host polymers may result in a reduced effectiveness of CNTs as material reinforcement.

Stress transfer from polymer matrix to nanotubes is considered as a main reinforcement mechanism in CNT-reinforced composites. Therefore the elastic properties of CNT-reinforced polymers can be modelled using composite theory developed for short fibre-reinforced polymers, ${ }^{4,28}$ which defines the modulus of the composite as a function of the elastic properties of the fibres and matrix, together with the aspect ratio of the fibres and their alignment relative to the applied stress. For randomly aligned MWCNTs, the elastic modulus of the composite $\left(E_{c}\right)$ can be predicted by the Halpin-Tsai equation: ${ }^{4,35}$ 


$$
\begin{aligned}
& \frac{E_{c}}{E_{m}}=\frac{3}{8}\left(\frac{1+\zeta \eta_{L} V_{f}}{1-\eta_{L} V_{f}}\right)+\frac{5}{8}\left(\frac{1+2 \zeta \eta_{T} V_{f}}{1-\eta_{T} V_{f}}\right) \\
& \zeta=\frac{2 l}{d} \\
& \eta_{L}=\frac{E_{f} / E_{m}-1}{E_{f} / E_{m}+\zeta} \\
& \eta_{T}=\frac{E_{f} / E_{m}-1}{E_{f} / E_{m}+2}
\end{aligned}
$$

where $E_{m}$ is the elastic modulus of the polymer matrix, and $l$ is the average length of the nanotubes. $V_{f}$ represents the volume fraction of the nanotubes, which can be calculated from

$$
V_{f}=\frac{1}{1+\frac{\rho_{C N T}}{\rho_{m}}\left(\frac{1}{W_{f}}-1\right)}
$$

where $\rho_{m}$ is the density of the polymer matrix (for PPS, $\rho_{m}=1350 \mathrm{~kg} / \mathrm{m}^{3}$ ). ${ }^{36}$

The elastic modulus of each PPS nanocomposite predicted using Equations $(2,4-8)$ and the distribution of nanotube diameters (Table 1) exhibited good agreement with the measured results at low CNT loadings (below $1 \mathrm{wt} \%$ ), as shown in Figure 4. However, the predicted modulus began to exceed the experimental data at CNT loadings higher than $1 \mathrm{wt} \%$. And the divergence between the predicted and measured values becomes larger with increasing CNT content. This overestimation was also reported in high density polyethylene reinforced with Nanocyl MWCNTs of various types (including NC 7000). ${ }^{27}$ It is intriguing since the Halpin-Tsai equation is usually known to fit the real modulus very well at low CNT loadings but to give underestimated results at high CNT loadings. ${ }^{4}$ Therefore, the decrease in reinforcing efficiency of MWCNTs at high CNT loadings should be taken into account in the model. An efficiency 
factor $\left(F\left(V_{f}\right)\right)$ as a function of CNT volume fraction was introduced to modify the Halpin-Tsai equation:

$$
E_{c}=F\left(V_{f}\right)\left[\frac{3}{8}\left(\frac{1+\zeta \eta_{L} V_{f}}{1-\eta_{L} V_{f}}\right)+\frac{5}{8}\left(\frac{1+2 \zeta \eta_{T} V_{f}}{1-\eta_{T} V_{f}}\right)\right] E_{m}
$$

The analysis of data revealed that $F\left(V_{f}\right)$ had a linear correlation to $V_{f}$ and can be expressed by

$$
F\left(V_{f}\right)=1+\alpha V_{f}
$$

where $\alpha=-4.46$.

The modulus predicted using the modified model compares well with the experimental data, as shown in Figure 4. To verify this model further, we applied it to the published experimental results of PEEK nanocomposites filled with NC 7000 MWCNTs and prepared by twin-screw extrusion. ${ }^{31}$ The calculated modulus was close to the measured values in the CNT content range from 2 to $10 \mathrm{wt} \%$ (Figure 5). However, both the original and modified Halpin-Tsai models gave a considerable deviation from the experimental result at a very high CNT loading (17 wt\%), indicating that other factors likely act as a pivotal role in the mechanism of reinforcement at that loading level.

\subsection{Crystallization and melt behaviour of PPS/MWCNT composites}

The addition of MWCNTs demonstrated an unconventional effect on the crystallization of PPS. Figure 6a shows the cooling DSC thermograms of PPS/MWCNT composites. The peak crystallization temperature $\left(T_{c}\right)$ of PPS and its nanocomposites was around $253{ }^{\circ} \mathrm{C}$. In the CNT content range between 0.5 and $4.0 \mathrm{wt} \%$, the onset crystallization temperature $\left(T_{c \text {-onset }}\right)$ remained at the same level (about $269^{\circ} \mathrm{C}$ ). But a distinct shift (about $10{ }^{\circ} \mathrm{C}$ ) of the ending temperature $\left(T_{c \text { - }}\right.$ end) towards the lower end was observed in the nanocomposites (as listed in Table 3), indicative 
of an extended crystallization process. According to the DSC data, the time that the PPS nanocomposites took for a complete crystallization process was about 100 seconds longer than the unfilled PPS. The crystallization of PPS with $8.0 \mathrm{wt} \%$ MWCNTs started slightly earlier (at $271{ }^{\circ} \mathrm{C}$ ), and lasted as long as the other nanocomposites. A discernible shoulder can be seen on the left side of the crystallization peak (around $232{ }^{\circ} \mathrm{C}$ ) when the CNT content exceeds $4.0 \mathrm{wt} \%$. The degree of crystallinity of the nanocomposites exhibited an unusual tendency. The $\chi_{c}$ was found to reach its peak value at CNT loading of $0.5 \mathrm{wt} \%, 12 \%$ higher than that of unfilled PPS. However, additional increases in CNT content gave no further rise but a small reduction in the crystallinity of PPS nanocomposites, as compared to the highest level.

Carbon nanotubes have been extensively reported to act as nucleating agents and to accelerate the crystallization rate. ${ }^{37-39}$ However, our observations show that MWCNTs have a dual effect on the crystallization of PPS. In addition to promoting nucleation, MWCNTs can also impose a confinement on the movement of polymer chains and hamper crystal growth. ${ }^{40,41}$ The two effects competed against each other during the crystallization process, leading to a dependency of the crystallization behaviour of PPS on the MWCNT weight fraction. On one hand, the additional sites present on the surface of CNTs can promote the formation of more crystalline structure and make the crystallization initiate earlier at high CNT loading (e.g. $8 \mathrm{wt} \%$ ). On the other hand, the organisation of polymer chains into ordered crystalline arrangements was suppressed by the network formed by the MWCNTs within the polymer matrix, hence an extended crystallization process was observed in PPS nanocomposites. The promotion effect has the initiative in this competition at low CNT loadings (e.g. $0.5 \mathrm{wt} \%$ ), leading to a significant increase in the degree of crystallinity. Nevertheless, the confinement effect became more and more dominant with increasing CNT loading, resulting in a slight decrease in crystallinity. 
The melting behaviour of PPS nanocomposites was almost unchanged with the presence of MWCNTs, as shown in the DSC thermograms measured during the second heating stage (Figure 6b). In all six systems, the peak melting temperature $\left(T_{m}\right)$ was about $284{ }^{\circ} \mathrm{C}$, and both the onset and end points remained similar (Table 3).

\section{Conclusion}

This work documents the preparation of PPS/MWCNT composites through a specialized melt-blending procedure. An efficient dispersion of the nanotubes in the PPS matrix with loadings in the range from 0.5 to $8.0 \mathrm{wt} \%$ was achieved by combining twin-screw extrusion with centrifugal pre-mixing.

MWCNTs showed a significant reinforcing effect on the mechanical properties of PPS. Increases up to $36 \%$ and $14 \%$ were observed in modulus and strength respectively. However, the magnitude of this effect was still less than expected, considering the superior mechanical performance of CNTs. The reinforcement efficiency of MWCNT was found to decrease at high CNT loadings. Based on this observation, the Halpin-Tsai equation was modified by an efficiency factor, which was linearly related to CNT volume fraction. This theoretical model was found effective to predict the elastic modulus of MWCNT-reinforced high performance thermoplastics, including PPS and PEEK (using published experimental data), over a specified range of CNT loadings.

The incorporation of MWCNTs exhibited both promotion and retardation effects on the crystallization in PPS. The former, prevalent at low CNT contents, led to an increase in the degree of crystallinity, whereas the latter extended the crystallization process and resulted in less overall crystallinity at high CNT contents. These two competing roles yielded a dependency of 
crystallization behaviour on CNT content, manifesting itself in an unusual change of the degree of crystallinity in PPS nanocomposites as a function of CNT weight fraction. The melting behaviour of PPS was nearly not affected by MWCNTs, which could be a benefit from an industrial point of view since no additional energy will be required in the further processing of PPS/MWCNT composites when using them as matrix materials for fabrication of advanced fibre-reinforced composites, which are expected to benefit from the enhanced mechanical properties of PPS/MWCNT composites.

\section{Acknowledgements}

The authors are grateful to the financial support provided by the Department for Employment and Learning, Northern Ireland, through the 'Sustainable Transport' project under the 'Strengthening the All-Island Research Base' Programme. The authors would like to thank Mr J. Kissick, Dr. P. Hanna and Dr. S. McFarland at Queen's University Belfast for their kind assistance in twin-screw extrusion, injection moulding and SEM characterization, respectively.

\section{Reference}

1 Thostenson, E. T.; Ren, Z.; Chou, T.-W. Compos Sci Technol 2001, 61, 1899.

2 Baughman, R. H.; Zakhidov, A. A.; de Heer, W. A. Science 2002, 297, 787.

3 Moniruzzaman, M.; Winey, K. I. Macromolecules 2006, 39, 5194.

4 Coleman, J. N.; Khan, U.; Blau, W. J.; Gun'ko, Y. K. Carbon 2006, 44, 1624.

5 Qian, H.; Greenhalgh, E. S.; Shaffer, M. S. P.; Bismarck, A. J Mater Chem 2010, 20, 4751.

6 Chou, T. W.; Gao, L.; Thostenson, E. T.; Zhang, Z.; Byun, J. H. Compos Sci Technol 2010, 70, 1 . 
7 Platt, D. K. Engineering and High Performance Plastics; Rapra Technology Ltd: Shawbury, 2003.

8 Schwartz, C. J.; Bahadur, S. Wear 2000, 237, 261.

9 Lu, D.; Yang, Y.; Zhuang, G.; Zhang, Y.; Li, B. Macromol Chem Phys 2001, 202, 734.

10 Lu, D.; Mai, Y.-w.; Li, R. K. Y.; Ye, L. Macromol Mater Eng 2003, 288, 693.

11 Bahadur, S.; Sunkara, C. Wear 2005, 258, 1411.

12 Cho, M. H.; Bahadur, S.; Pogosian, A. K. Tribol Int 2006, 39, 249.

13 Lu, D.; Pan, S. Polym Eng Sci 2006, 46, 820.

14 Wang, X.; Tong, W.; Li, W.; Huang, H.; Yang, J.; Li, G. Polym Bull 2006, 57, 953.

15 Naffakh, M.; Marco, C.; Gomez, M. A.; Jimenez, I. J Phys Chem B 2008, 112, 14819.

16 Naffakh, M.; Marco, C.; Gomez, M. A.; Gomez-Herrero, J.; Jimenez, I. J Phys Chem B 2009, 113, 10104.

17 Sugama, T. Mater Lett 2006, 60, 2700

18 Zou, H.; Xu, W.; Zhang, Q.; Fu, Q. J Appl Polym Sci 2006, 99, 1724.

19 Zhao, Y. F.; Xiao, M.; Wang, S. J.; Ge, X. C.; Meng, Y. Z. Compos Sci Technol 2007, 67, 2528.

20 Cho, M. Mater Trans 2008, 49, 2801.

21 Yang, J.; Xu, T.; Lu, A.; Zhang, Q.; Fu, Q. J Appl Polym Sci 2008, 109, 720.

22 Yu, S.; Wong, W. M.; Hu, X.; Juay, Y. K. J Appl Polym Sci 2009, 113, 3477.

23 Wu, D.; Wu, L.; Zhou, W.; Yang, T.; Zhang, M. Polym Eng Sci 2009, 49, 1727.

24 Yang, J.; Xu, T.; Lu, A.; Zhang, Q.; Tan, H.; Fu, Q. Compos Sci Technol 2009, 69, 147.

25 Lopez, L. C.; Wilkes, G. L. Polym-plast Technol 1989, 29, 83.

26 Mark, J. E. Polymer Data Handbook; Oxford University Press: New York, 1999.

27 Morcom, M.; Atkinson, K.; Simon, G. P. Polymer 2010, 51, 3540.

28 Thostenson, E. T.; Chou, T.-W. Journal of Physics D: Applied 2003, 36, 573.

29 Cadek, M.; Coleman, J. N.; Barron, V.; Hedicke, K.; Blau, W. J. Appl Phys Lett 2002, 81, 5123. 
30 Cebe, P. Polym Polym Compos 1995, 3, 239.

31 Bangarusampath, D. S.; Ruckdaschel, H.; Altstadt, V.; Sandler, J. K. W.; Garray, D.; Shaffer, M. S. P. Polymer 2009, 50, 5803.

32 Byrne, M. T.; Gun'ko, Y. K. Advanced materials (Deerfield Beach, Fla.) 2010, 22, 1672.

33 Hussain, F.; Hojjati, M.; Okamoto, M.; Gorga, R. E. J Compos Mater 2006, 40, 1511.

34 Rong, C.; Ma, G.; Zhang, S.; Song, L.; Chen, Z.; Wang, G.; Ajayan, P. M. Compos Sci Technol 2010, 70, 380 .

35 Halpin, J. C.; Kardos, J. L. Polym Eng Sci 1976, 16, 344.

36 www.ticona.com Fortron PPS Datasheet.

37 Assouline, E.; Lustiger, A.; Barber, A. H.; Cooper, C. A.; Klein, E.; Wachtel, E.; Wagner, H. D. J Polym Sci Pol Phys 2002, 41, 520.

38 Bhattacharyya, A. R.; Sreekumar, T. V.; Liu, T.; Kumar, S.; Ericson, L. M.; Hauge, R. H.; Smalley, R. E. Polymer 2003, 44, 2373.

39 Haggenmueller, R.; Fischer, J. E.; Winey, K. I. Macromolecules 2006, 39, 2964.

40 Li, L.; Li, C.; Ni, C.; Rong, L.; Hsiao, B. Polymer 2007, 48, 3452.

41 Díez-Pascual, A. M.; Naffakh, M.; Gómez, M. A.; Marco, C.; Ellis, G.; Martínez, M. T.; Ansón, A.; González-Domínguez, J. M.; Martínez-Rubi, Y.; Simard, B. Carbon 2009, 47, 3079. 
Figures and tables

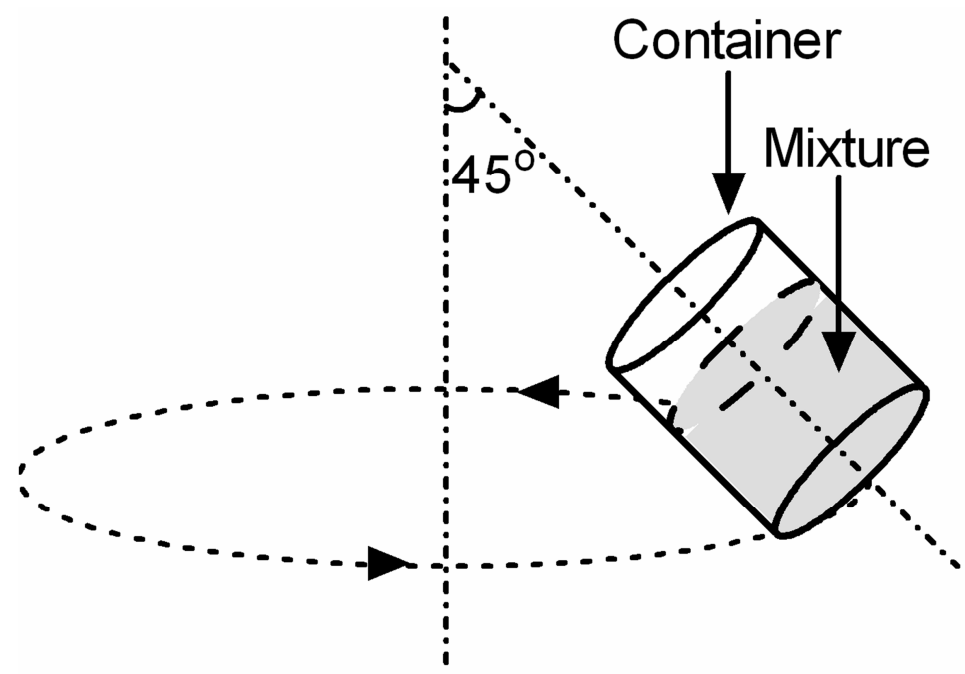

Figure 1. Illustration of centrifugal pre-mixing 

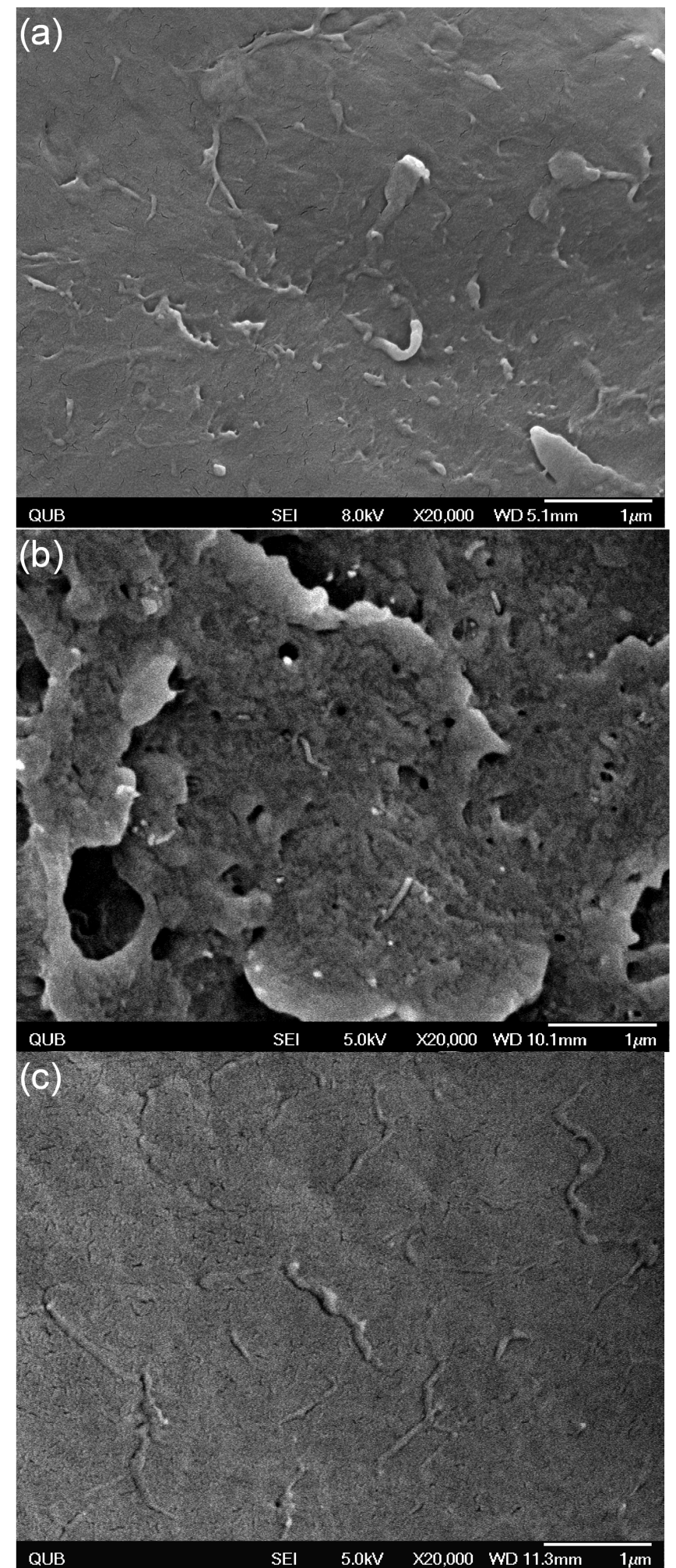

Figure 2. SEM micrographs of the cryo-fractured surfaces of (a) PPS with $0.5 \mathrm{wt} \%$ MWCNT, (b) PPS with $2.0 \mathrm{wt} \%$ MWCNT and (c) PPS with $8.0 \mathrm{wt} \%$ MWCNT.

Page 17 of 20 


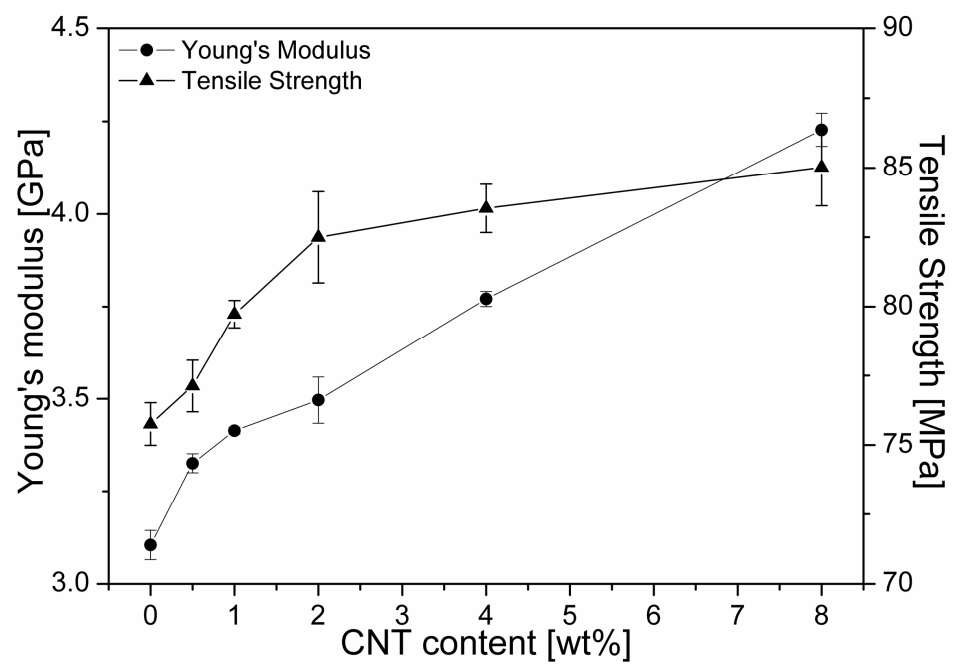

Figure 3. Mechanical properties of PPS/MWCNT composites

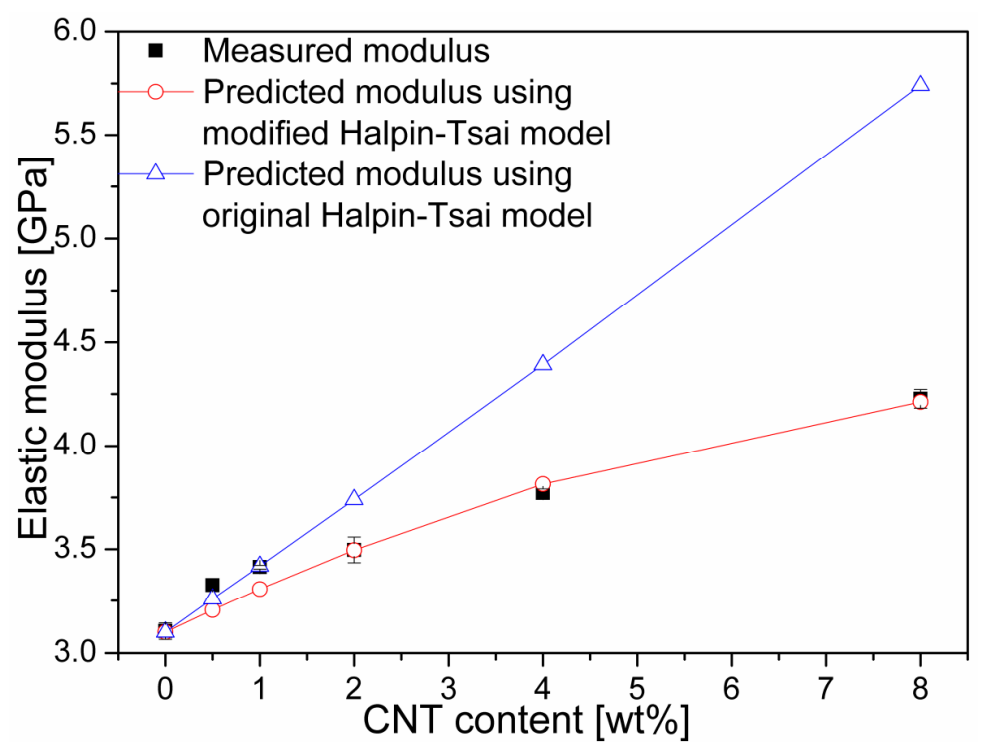

Figure 4. Prediction of elastic modulus of PPS/MWCNT composites using Halpin-Tsai equations. 


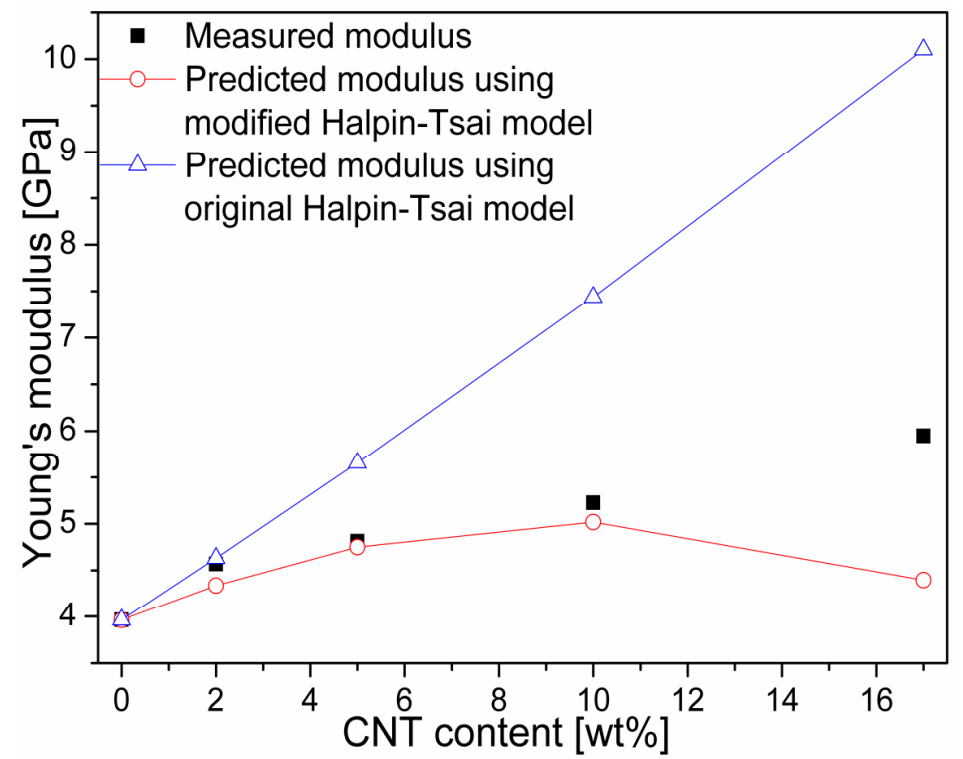

Figure 5. Prediction of elastic modulus of PEEK/MWCNT composites using Halpin-Tsai equations. The experimental values were obtained from Ref. ${ }^{31}$.
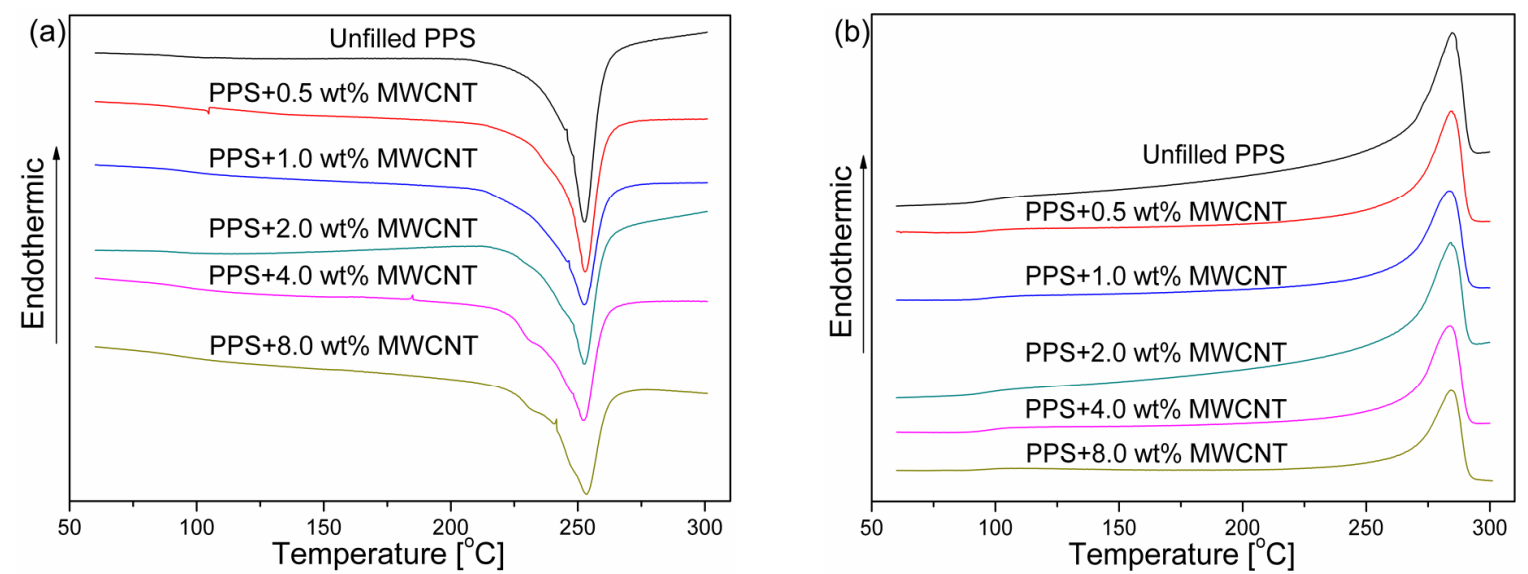

Figure 6. Non-isothermal DSC thermograms of PPS/MWCNT composites at (a) $1^{\text {st }}$ cooling stage and (b) $2^{\text {nd }}$ heating stage.

Table 1. Statistical distribution of nanotube diameter for Nanocyl NC 7000. Reproduced from Ref. ${ }^{27}$. \begin{tabular}{llllllllllllllllll} 
CNT diameter $[\mathrm{nm}]$ & 5 & 6 & 7 & 8 & 9 & 10 & 11 & 12 & 13 & 14 & 15 & 17 & 18 & 19 & 20 & 22 & 23 \\
\hline
\end{tabular} 


\begin{tabular}{llllllllllllllllll}
\hline Frequency [\%] & 1 & 5 & 10 & 3 & 14 & 16 & 14 & 15 & 6 & 4 & 4 & 2 & 1 & 1 & 2 & 1 & 1 \\
\hline
\end{tabular}

Table 2. Mechanical properties of PPS/MWCNT composites

\begin{tabular}{llllll}
\hline $\begin{array}{l}\text { MWCNT } \\
\text { content } \\
{[\mathrm{wt} \%]}\end{array}$ & $\begin{array}{l}\text { Young's } \\
\text { modulus } \\
{[\mathrm{GPa}]}\end{array}$ & $\begin{array}{l}\text { Tensile } \\
\text { strength } \\
{[\mathrm{MPa}]}\end{array}$ & $\begin{array}{l}\text { Breaking } \\
\text { strain } \\
{[\%]}\end{array}$ & $\begin{array}{l}\text { Flexural } \\
\text { modulus } \\
{[\mathrm{GPa}]}\end{array}$ & $\begin{array}{l}\text { Flexural } \\
\text { strength } \\
{[\mathrm{MPa}]}\end{array}$ \\
\hline 0 & $3.10 \pm 0.04$ & $75.75 \pm 0.77$ & $5.97 \pm 0.68$ & $3.48 \pm 0.07$ & $88.15 \pm 1.20$ \\
0.5 & $3.33 \pm 0.03$ & $77.13 \pm 0.93$ & $5.44 \pm 0.30$ & $3.32 \pm 0.05$ & $88.07 \pm 3.54$ \\
1 & $3.41 \pm 0.01$ & $79.73 \pm 0.50$ & $5.01 \pm 0.70$ & $3.56 \pm 0.06$ & $88.52 \pm 1.29$ \\
2 & $3.50 \pm 0.06$ & $82.50 \pm 1.64$ & $3.69 \pm 0.60$ & $3.58 \pm 0.09$ & $92.36 \pm 2.76$ \\
4 & $3.77 \pm 0.02$ & $83.54 \pm 0.87$ & $3.57 \pm 0.46$ & $3.72 \pm 0.07$ & $94.00 \pm 1.87$ \\
8 & $4.23 \pm 0.04$ & $85.03 \pm 1.40$ & $3.23 \pm 0.23$ & $4.18 \pm 0.15$ & $100.11 \pm 1.76$ \\
\hline
\end{tabular}

Table 3. Crystallization and melting data of PPS/MWCNT composites obtained through DSC

\begin{tabular}{llllllll}
\hline $\begin{array}{l}\text { MWCNT } \\
\text { content } \\
{[\mathrm{wt} \%]}\end{array}$ & $\begin{array}{l}T_{c} \\
{\left[{ }^{\circ} \mathrm{C}\right]}\end{array}$ & $\begin{array}{l}T_{\text {c-onset }} \\
{\left[{ }^{\circ} \mathrm{C}\right]}\end{array}$ & $\begin{array}{l}T_{c \text {-end }} \\
{\left[{ }^{\circ} \mathrm{C}\right]}\end{array}$ & $\begin{array}{l}\chi \\
{[\%]}\end{array}$ & $\begin{array}{l}T_{m} \\
{\left[{ }^{\circ} \mathrm{C}\right]}\end{array}$ & $\begin{array}{l}T_{m \text {-onset }} \\
{\left[{ }^{\circ} \mathrm{C}\right]}\end{array}$ & $\begin{array}{l}T_{m \text {-end }} \\
{\left[{ }^{\circ} \mathrm{C}\right]}\end{array}$ \\
\hline 0 & $252.75 \pm 0.08$ & $268.37 \pm 0.75$ & $226.58 \pm 0.09$ & $32.76 \pm 2.49$ & $285.03 \pm 0.50$ & $252.30 \pm 0.35$ & $295.59 \pm 0.86$ \\
0.5 & $252.69 \pm 0.37$ & $269.18 \pm 2.09$ & $216.38 \pm 0.94$ & $36.76 \pm 1.78$ & $284.69 \pm 0.65$ & $251.61 \pm 0.68$ & $295.38 \pm 1.17$ \\
1 & $252.62 \pm 0.11$ & $268.38 \pm 0.95$ & $216.72 \pm 0.66$ & $35.48 \pm 1.22$ & $284.05 \pm 0.93$ & $250.27 \pm 0.27$ & $295.97 \pm 2.01$ \\
2 & $252.21 \pm 0.46$ & $268.17 \pm 1.99$ & $217.51 \pm 0.11$ & $35.00 \pm 0.72$ & $284.41 \pm 0.44$ & $251.50 \pm 1.10$ & $295.73 \pm 0.37$ \\
4 & $252.21 \pm 0.28$ & $269.71 \pm 2.26$ & $216.91 \pm 0.37$ & $36.09 \pm 1.26$ & $284.25 \pm 0.35$ & $251.28 \pm 0.47$ & $295.51 \pm 1.03$ \\
8 & $252.95 \pm 0.75$ & $271.18 \pm 1.12$ & $219.31 \pm 0.76$ & $35.37 \pm 0.12$ & $284.31 \pm 0.24$ & $252.50 \pm 0.44$ & $295.72 \pm 0.48$ \\
\hline
\end{tabular}

